\title{
Interleukin-17, produced by lymphocytes, promotes tumor growth and angiogenesis in a mouse model of breast cancer
}

\author{
JIU-WEI DU, KE-YI XU, LI-YI FANG and XIN-LAN QI \\ School of Medicine, Anhui University of Science and Technology, Huainan, Anhui 232001, P.R. China
}

Received April 7, 2012; Accepted August 2, 2012

DOI: $10.3892 / \mathrm{mmr} .2012 .1036$

\begin{abstract}
Previous studies have suggested that interleukin-17 (IL-17), an inflammatory cytokine expressed predominantly by Th17 cells, is highly expressed in tumor tissue and may help tumors to evade immune surveillance. In this study, the significance of IL-17 expression in the tumors of murine models of breast cancer was explored. BALB/c mice were injected with MA782/5S28102 or 4T1 breast cancer cell lines to establish breast tumors. The expression of IL-17 in tumor tissue was detected by western blotting 1 and 4 weeks later, which revealed that it increased with tumor progression $(\mathrm{P}<0.05)$. Additionally, tumor cells and tumor-infiltrating lymphocytes were isolated from tumor tissues and cultured for 5 days with stimulation by phorbol-12-myristate-13-acetate (PMA), anti-CD3 antibody and anti-CD28 antibody. Culture media from stimulated tumor cells or tumor-infiltrating lymphocytes were harvested and their concentrations of IL-17 were tested by ELISA. Tumor cells secreted low levels of IL-17 into the media; however, lymphocytes from tumor tissues secreted high levels of IL-17, with 4T1 tumors secreting higher levels of IL-17 than MA782 tumors $(\mathrm{P}<0.05)$. To evaluate the effect of IL-17 on the proliferation of tumor cells, 4T1 cells were cultured in the presence or absence of recombinant IL-17 and cell numbers were counted on day 5 of culturing. Ectopic IL-17 did not promote the proliferation of tumor cells in vitro. To further understand the effect of IL-17 expression within tumors, 4T1 tumor-bearing mice were injected with recombinant IL-17 or saline via the tail vein. Tumor size was measured up to 21 days following the initial infusion of IL-17. IL-17 infusion resulted in an increased tumor volume and microvascular density (as measured by the immunohistochemical detection of CD34 expression in microvessels; $\mathrm{P}<0.05$ ). Therefore, IL-17 expression within tumor tissues appears to originate from
\end{abstract}

Correspondence to: Professor Jiu-Wei Du, School of Medicine, Anhui University of Science and Technology, 25 Dongshanzhong Road, Huainan, Anhui 232001, P.R. China

E-mail: djwdxswy@126.com

Key words: breast cancer, mouse model, interleukin-17, angiogenesis tumor-infiltrating lymphocytes and is likely to promote tumor growth by enhancing angiogenesis.

\section{Introduction}

Breast cancer occurs in approximately 99.4 of every 100,000 women in North America and is the most frequent malignancy in women $(1,2)$. The disease is the second leading cause of mortality due to cancer among women $(3,4)$. Given its incidence and threat to survival rate, much effort has been devoted to understanding the etiology of breast tumors. Numerous discoveries have arisen with regard to the genetic and molecular origins and progression of this disease. However, breast tumor cells are, in some cases, able to evade immune surveillance, allowing the tumor to grow and advance.

The mechanisms by which these tumor cells escape detection remain unknown; however, previous research has confirmed that interleukin-17 (IL-17) is significant in promoting tumor growth (5-7). IL-17 is an inflammatory cytokine that has functions in innate and adaptive immunity and is expressed from Th17 cells (8). However, the dysregulation of IL-17 is apparent in breast tumors, demonstrated by the presence of IL-17-expressing cells within them. This indicates that IL-17 aids these cells in escaping elimination by immune cells, thereby promoting tumor progression (9).

In this study, murine breast cancer models were established to analyze the expression of IL-17 within mammary tumors. The correlation between IL-17 expression and tumor development was investigated.

\section{Materials and methods}

Establishing mouse breast cancer models. Female BALB/c mice, 6-8 weeks old, average body weight $20.5 \pm 2.4 \mathrm{~g}$, were purchased from the Experimental Animal Center of Shanghai Second Military Medical University (Shanghai, China). Mouse breast cancer cell lines MA782 (MA782/5S28102, China Center for Type Culture Collection, Wuhan University, Hubei, China) and 4T1 (American Type Culture Collection, Manassas, VA, USA) were cultured in RPMI-1640 medium supplemented with $100 \mathrm{U} / \mathrm{ml}$ penicillin, $100 \mathrm{U} / \mathrm{ml}$ streptomycin and $2 \mathrm{M}$ glutamine at $37^{\circ} \mathrm{C}$ and $5 \% \mathrm{CO}_{2}$. The cells were harvested during the logarithmic growth phase. Mice (32) were randomly divided into four groups of 8 . Mice in two groups received subcutaneous injections of MA782 cells 
$\left(1 \times 10^{6}\right.$ cells/mouse) into the mammary gland on the left abdominal wall; mice in the other two groups received injections of $4 \mathrm{~T} 1$ cells $\left(5 \times 10^{6}\right.$ cells/mouse). A tumor $2 \times 2 \mathrm{~mm}$ in size was considered to be a successfully established model. The study was approved by the ethics committee of the School of Medicine, Anhui University of Science and Technology.

Lymphocyte and tumor cell collection from tumor samples. Inoculated mice were sacrificed by decapitation at tumor-bearing weeks 1 (early) and 4 (late). Tumor tissues were excised and minced ( $<3 \mathrm{~mm}^{3}$ pieces), washed with phosphate-buffered saline (PBS), then digested in calf serum-free medium with $0.05 \%$ collagenase IV, $100 \mathrm{mg} / \mathrm{l}$ DNase I (Sigma, St. Louis, MO, USA) and $16 \mathrm{M} \mathrm{CaCl}_{2}$. Tissue pieces were oscillated for $40 \mathrm{~min}$ in digestion buffer, then re-suspended in mouse lymphocyte separation medium. The upper layer was covered with a thin layer of RPMI-1640. Following gradient centrifugation, the lymphocyte layer was collected and washed, then re-suspended in culture medium to isolate tumor-infiltrating lymphocytes; the lymphocyte separation medium and sediment layers were collected and washed, then re-suspended in culture medium to isolate tumor cells.

Western blotting. Tumor tissue was homogenized and treated with $100 \mathrm{ml}$ cell lysis buffer on ice for $30 \mathrm{~min}$. Samples were centrifuged at $4^{\circ} \mathrm{C}$ and $12,000 \mathrm{x}$ g for $15 \mathrm{~min}$. Supernatant was transferred to another centrifuge tube for the determination of total protein content using the Coomassie brilliant blue microdisk (Sigma) colorimetric method. Total protein was subjected to SDS-PAGE for western blotting to detect IL-17 expression with anti-IL-I7 monoclonal antibodies (Santa Cruz Biotechnology, Inc., Santa Cruz, CA, USA) and HRP-labeled secondary antibodies. Following detection of bands on nitrocellulose membranes, Quantity One (BioRad, Hercules, CA, USA) was used to analyze the gray-value of the target band. Relative expression levels of the target band were compared to the $\beta$-actin control.

ELISA. Phorbol-12-myristate-13-acetate (PMA), anti-CD3 monoclonal antibody and anti-CD28 monoclonal antibody were added to lymphocyte and tumor cell cultures isolated from tumor tissue and continuously cultured for 5 days. ELISA kits were used to detect IL-I7 expression (R\&D Systems, Minneapolis, MN, USA) in supernatants according to the manufacturer's instructions.

IL-17 infusion and tumor growth. Recombinant IL-17 (50 $\mu \mathrm{g} / \mathrm{l}$, Pepro Tech, Rocky Hill, NJ, USA) was added to 4T1 cells in culture; the cell proliferation rate was analyzed after 5 days. $\mathrm{BALB} / \mathrm{c}$ mice (16) were inoculated with treated $4 \mathrm{~T} 1$ cells, then randomly divided into infusion and non-infusion groups. Mice in the infusion group received injections of $1 \mu \mathrm{g}$ IL-17 via the caudal vein on days 1, 7 and 14; mice in the non-infusion group received saline only on those days. Tumor size and volumes were measured [tumor volume $=\left(\right.$ length $\mathrm{x}$ short diameter $\left.{ }^{2}\right) / 2$ ]

Detection of CD34 expression by immunohistochemistry. Tissues were fixed in neutral formalin, dehydrated and embedded in paraffin wax for sectioning (slice thickness of $4 \mu \mathrm{m}$ ) by conventional methods. Sections were dewaxed with dimethylbenzene and rehydrated, then heated for antigen retrieval. Endogenous peroxidase activity was blocked by treatment with 3\% hydrogen peroxide solution. Sections were then sealed with non-specific serum and placed in a wet box at room temperature. Primary antibodies (Mena and Her-2) were added to the wet box for incubation at $4^{\circ} \mathrm{C}$ overnight. Following incubation, sections were washed with PBS three times, then treated with biotinylated secondary antibodies and incubated at room temperature. Following three more washes in PBS, streptococcus-avidin-peroxidase (Zhongshang Golden Bridge Biotechnology Co., Ltd., Beijing, China) was added to the wet box and sections were incubated at $37^{\circ} \mathrm{C}$ for $30 \mathrm{~min}$. Staining was developed with DAB chromogen (Dako, Carpinteria, CA, USA) and detected under a light microscope. Known positive tissue sections were used as positive controls and PBS was used in place of primary antibodies as a negative control. CD34 staining appears as brownish-yellow or sepia-toned endothelial cell membranes or cytoplasm. Neovascularization is usually expressed as tumor microvascular density (MVD), as reported by Weidner (10). The region with the most dense microvascular distribution under x100 light microscopy was assessed by counting the number of vessels expressing CD34 in 5 individual visual fields. The mean value is expressed as MVD in units of count at x200 magnification. Positively-stained single endothelial cells or endothelial cell clusters that were significantly separated from the neighboring microvessels were counted; all blood vessels with lumens greater than 8 red blood cells and a thicker muscular layer were not counted.

Statistical analysis. SPSS 17.0 software was used for statistical analysis. Data are expressed as mean \pm SEM. The analysis was performed with a two-sided test, with the $\alpha$ level equal to 0.05 and $\mathrm{P}<0.05$ considered to indicate statistically significant differences.

\section{Results}

IL-17 expression in tumoral tissue. To determine whether IL-17 expression is dysregulated in breast tumors, tumor tissues from mice inoculated with MA782 or 4T1 breast cancer cells were subjected to western blotting. IL-17 expression was detected in tumor samples from mice inoculated with MA782 cells and 4T1 cells, as well as in early and late stage tumors. However, the expression levels of IL-17 were significantly higher in late than in early stage tumor tissues (P<0.05; Fig. 1).

IL-17 is expressed by CD4 ${ }^{+} \mathrm{T}$ helper cells, namely Th17 cells $(6,11)$. To determine whether the tumor cells were expressing IL-17, or whether other IL-17-expressing cells had infiltrated the tumors, IL-17 expression levels from cell cultures in which the breast cancer cells had been separated from lymphocytes in the tumor tissue were assessed. After 5 days in separate cultures, the concentrations of IL-17 in the supernatants from each culture were tested by ELISA. Low levels of IL-17 were secreted into the supernatant by tumor cells; however, lymphocytes from the tumor tissues secreted a higher level of IL-17. A higher level of IL-17 was detected in the supernatant of lymphocytes from tumors of mice inoculated with 4T1 cells than those inoculated with MA782 $(\mathrm{P}<0.05$; Fig. 2). 


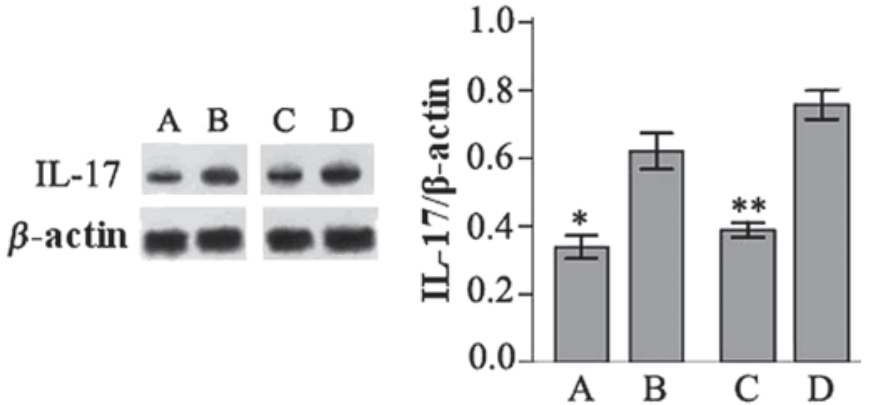

Figure 1. IL-17 expression in tumor tissues of mice inoculated with two different breast cancer cell lines. BALB/c female mice were injected with breast cancer tumor cell lines (A and B) MA782 or (C and D) 4T1 in the abdominal wall. IL-17 expression was detected within tumor tissues by western blotting at either (A and C) early stages (1 week) or (B and D) late stages (4 weeks) of tumor development. ${ }^{*} \mathrm{P}<0.05$ vs. $\mathrm{B}$ group; ${ }^{* *} \mathrm{P}<0.05$ vs. $\mathrm{D}$ group. IL-17, interleukin-17.

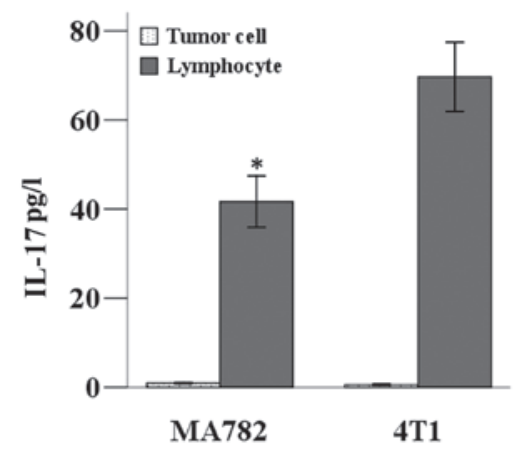

Figure 2. IL-17 expression in separated tumor cells and lymphocytes after placement in culture. Tumor cells and lymphocytes were separated from tumor tissues of mice inoculated with MA782 or 4T1 cells. After 5 days in culture, supernatants were assayed by ELISA for concentration of IL-17. ${ }^{*} \mathrm{P}<0.05$ vs. 4T1 group. Both types of tumor cells secreted little IL-17 into the supernatant, but lymphocytes from both tumor tissues secreted high levels of IL-17. IL-17, interleukin-17.

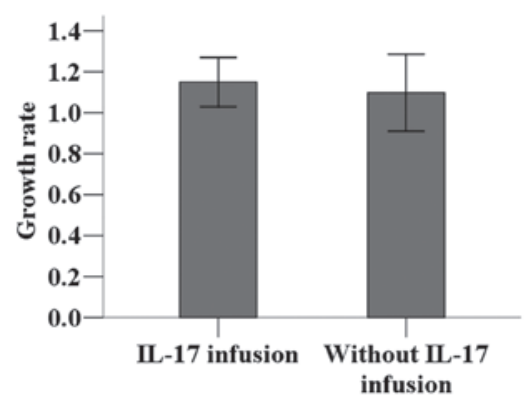

Figure 3. Effect of IL-17 infusion on tumor cell proliferation. 4T1 breast cancer cells were cultured for 5 days in the presence or absence of recombinant IL-17, then cells were counted. IL-17 in culture medium did not affect tumor cell proliferation. IL-17, interleukin-17.

Effect of IL-17 infusion on proliferation of tumor cells. The 4T1 breast cancer cell line was cultured with or without recombinant IL-17 to determine its effect on tumor cell proliferation. No statistically significant difference was observed in the tumor cell proliferation rate between culture systems with and without IL-17 ( $\mathrm{P}>0.05$; Fig. 3). 4T1 cells cultured in

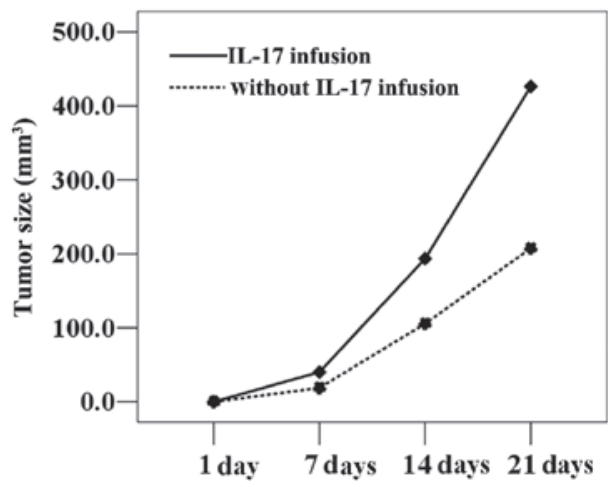

Figure 4. Effect of IL-17 infusion on size of tumors in mouse models. 4T1 cells were injected into abdominal walls of mice, which were then treated intravenously with either recombinant IL-17 or with saline only on days 1 , 7 and 14. Tumor size was measured on days 1, 7, 14 and 21. IL-17 infusion significantly increased tumor volume. IL-17, interleukin-17.

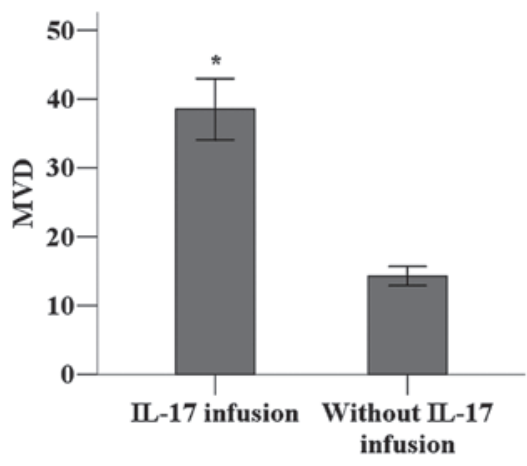

Figure 5. Effect of IL-17 infusion on MVD of tumors in mice inoculated with 4T1 cells. CD34 expression was used to determine MVD in tumor tissues of mice inoculated with $4 \mathrm{~T} 1$ cells and either receiving recombinant IL-17 infusion or saline only. Tumor tissue of mice receiving IL-17 infusion was significantly more vascularized. " $\mathrm{P}<0.05$ vs. without IL-17 infusion. IL-17, interleukin-17; MVD, microvascular density.

the presence of IL-17 were injected into BALB/c mice, which were then divided into infusion and non-infusion groups. The infusion group received injections of recombinant IL-17; the non-infusion groups received injections of saline only. Tumor growth was significantly accelerated in tumor-bearing mice following intravenous infusion of IL-17, resulting in significantly larger tumor volumes compared with those receiving saline only ( $\mathrm{P}<0.05$; Fig. 4).

Effects of IL-17 on angiogenesis in tumor tissues. The vascularization of tumor environments with higher levels of IL-17 was investigated. Immunohistochemistry was used to detect CD34 expression in tumor tissues to calculate MVD as a measure of angiogenesis. MVD was significantly higher in the tumor tissues of mice receiving IL-17 infusions compared with those receiving only saline infusions $(\mathrm{P}<0.05$; Fig. 5).

\section{Discussion}

IL-17 is a cytokine that is mainly secreted by Th17 cells during the inflammatory response $(6,11)$. Studies have shown that IL-17 promotes the release of IL-6, IL-8, prostaglandin E2 
(PGE2), IL- $1{ }^{\circledast}$, transforming growth factor (TNF) and some chemotactic factors, as well as the local aggregation of inflammatory cells (12). Previous studies have indicated that IL-17 is involved in the development and progression of numerous inflammatory diseases, including rheumatoid arthritis, systemic lupus erythematosus and certain respiratory diseases. Additionally, IL-17 is expressed at different levels in a number of tumors. Zhang et al (13) reported that IL-17 is present in the tumor tissues and serum of gastric cancer patients, with IL-17 expression levels correlating with the clinical stage of tumors. Similarly, the levels of IL-17 in the tumors of patients with colorectal cancer are significantly higher compared with those in normal tissues (14). In mouse models of non-small cell lung cancer, IL-17 transfection promotes tumor growth (15). Other studies suggest that IL-17 is involved in the development and progression of prostate, ovarian, choriocarcinoma and cutaneous T-cell tumors. The present study demonstrates that, in mouse breast cancer models, IL-17 expression is detected in tumor tissues at the early and late stages and IL-17 levels in late tumors are significantly higher compared with those in early tumors.

Shime et al (16) reported that tumor cells promote CD4 $4^{+}$ $\mathrm{T}$ cells to release IL-17 through the release of lactic acid. Kryczek et al (17) confirmed that IL-2 in the tumor environment regulates the balance between Treg cells and the secretion of IL-17. The present study revealed that IL-17 is mainly expressed by tumor-infiltrating lymphocytes, while tumor cells express little IL-17, indicating that IL-17 expression in tumors is the result of tumor microenvironment effects. However, it remains unclear how IL-17 expression in tumor tissues affects the occurrence and development of tumors. In the present study, in vitro exposure to IL-17 did not promote the proliferation of tumor cells; however, in vivo intravenous infusion of IL-17 in tumor-bearing mice significantly promoted tumor growth. This suggests that IL-17 indirectly promotes tumor growth. Furthermore, we demonstrated that vascular density significantly increased in tumors from mice receiving an intravenous infusion of IL-17. Therefore, IL-17 may accelerate the progression of tumors by promoting microvessel formation in tumor tissues.

In summary, in murine breast cancer models, tumor tissue expresses IL-17, with increasing expression levels in more advanced tumors. IL-17 may promote tumor growth by promoting microvessel formation in tumor tissues.

\section{Acknowledgements}

This study was supported by Science Fund of Anhui Educational Department (Grant No. KJ2012A080).

\section{References}

1. Wright SE: Immunotherapy of breast cancer. Expert Opin Biol Ther 12: 479-490, 2012.

2. World Health Organization. http://www.who.int/cancer/ detection/breastcancer/en/index1.html. Accessed April 2012.

3. Peng J, Sengupta S and Jordan VC: Potential of selective estrogen receptor modulators as treatments and preventives of breast cancer. Anticancer Agents Med Chem 9: 481-499, 2009.

4. Howard JH and Bland KI: Current management and treatment strategies for breast cancer. Curr Opin Obstet Gynecol 24: 44-48, 2012.

5. Novitskiy SV, Pickup MW, Gorska AE, et al: TGF- $\beta$ Receptor II loss promotes mammary carcinoma progression by Th17 dependent mechanisms. Cancer Discov 1: 430-441, 2011.

6. Afzali B, Lombardi G, Lechler RI and Lord GM: The role of T helper 17 (Th17) and regulatory T cells (Treg) in human organ transplantation and autoimmune disease. Clin Exp Immunol 148: 32-46, 2007.

7. Yang L, Qi Y, Hu J, Tang L, Zhao S and Shan B: Expression of Th17 cells in breast cancer tissue and its association with clinical parameters. Cell Biochem Biophys 62: 153-159, 2012.

8. Pappu R, Ramirez-Carrozzi V and Sambandam A: The interleukin-17 cytokine family: critical players in host defence and inflammatory diseases. Immunology 134: 8-16, 2011.

9. Zhu X, Mulcahy LA, Mohammed RA, et al: IL-17 expression by breast-cancer-associated macrophages: IL-17 promotes invasiveness of breast cancer cell lines. Breast Cancer Res 10: R95, 2008.

10. Weidner N: Current pathologic methods for measuring intratumoral microvessel density within breast carcinoma and other solid tumors. Breast Cancer Res Treat 36: 169-180, 1995.

11. Harrington LE, Haaon RD, Mangan PR, Turner H, Murphy TL, Murphy KM and Weaver CT: Interleukin 17-producing CD4+ effector $\mathrm{T}$ cells develop via a lineage distinct from the T helper type 1 and 2 lineages. Nat Immunol 6: 1123-1132, 2005.

12. Fossiez F, Banchereau J, Murray R, Van Kooten C, Garrone P and Lebecque S: Interleukin-17. Int Rev Immunol 16: 541-551, 1998.

13. Zhang B, Rong G, Wei H, Zhang M, Bi J, Ma L, Xue X, Wei G, Liu X and Fang G: The prevalence of Th17 cells in patients with gastric cancer. Biochem Biophys Res Commun 374: 533-537, 2008.

14. Le Gouvello S, Bastuji-Garin S, Aloulou N, et al: High prevalence of Foxp3 and IL-17 in MMR-proficient colorectal carcinomas. Gut 57: 772-779, 2008.

15. Numasaki M, Watanabe M, Suzuki T, et al: IL-17 enhances the net angiogenic activity and in vivo growth of human non-small cell lung cancer in SCID mice through promoting CXCR-2dependent angiogenesis. J Immunol 175: 6177-6189, 2005.

16. Shime H, Yabu M, Akazawa T, Kodama K, Matsumoto M, Seya $\mathrm{T}$ and Inoue N: Tumor-secreted lactic acid promotes IL-23/ IL-17 proinflammatory pathway. J Immunol 180: 7175-7183, 2008.

17. Kryczek I, Wei S, Zou L, Altuwaijri S, Szeliga W, Kolls J, Chang A and Zou W: Cutting ease: Thl7 and regulatory T cell dynamics and the regulation by IL-2 in the tumor microenvironment. J Immunol 178: 6730-6733, 2007. 